\title{
IMPLEMENTATION OF DYNAMIC MISALIGNMENTS AND LUMINOSITY STABILIZATION*
}

\author{
L. Hendrickson, T. Himel, T.O. Raubenheimer, A. Seryi, P. Tenenbaum, M. Woodley \\ SLAC, Stanford, USA \\ D. Schulte CERN, Geneva, Switzerland
}

\begin{abstract}
To fully characterize the luminosity performance of a linear collider, it is important to simulate the effects of ground motion as well as the beam-beam feedback and other stabilization systems planned to compensate for that motion. The linear collider simulation codes have recently been extended to include both ground motion models and stabilization systems to support the work of the International Linear Collider Technical Review Committee (TRC) [1]. This paper discusses the implementation details and the optimization strategies for interpulse beam-beam feedback.
\end{abstract}

\section{INTRODUCTION}

One of the TRC tasks was to evaluate strategies for keeping the beams in collision. Although simplified assumptions were used, the studies provide an initial look at operation of a linear collider and a basis for more detailed future work. For NLC, CLIC and TESLA, simulations included initial imperfections, realistic ground motion models, stabilization of the beam collisions at the interaction point (IP), and vibration of the final doublet. Results of these simulations are reported separately [2].

\section{Simulation Platform}

Many of the simulations were performed using MatlabLIAR [3], interfaced to Guinea-Pig [4] to simulate the beam-beam effects. MATLAB provides a flexible simulation platform, LIAR incorporates fast wakefield tracking with emittance dilution effects, and the DIMAD tracking engine is used for regions with nonlinear focusing elements, including the beam delivery, where wakefields are not a concern. A full ground motion simulation [5] is included, with diffusive ("ATL") motion as well as cultural noise. There are optional transfer functions at specified locations. In particular, vibration of the final doublet has a significant effect on the collisions at the IP, and it is important to include effects of additional vibration and any active stabilization [6].

In other simulations, the code PLACET [7] was used with Guinea-Pig. The code, which uses tcl-tk as an interface language, was extended to perform tracking in nonlinear elements and to allow the simulation of ground motion. A simplified modeling of the effect of slow feedbacks on the fast motion was incorporated.

\footnotetext{
* Work supported in part by US DOE, contract DE-AC0376 SF00515.
}

\section{Initialization of Imperfect Machine States}

In all proposed linear colliders, the beam emittance will increase during transport from damping rings to IP even after all means of emittance preservation have been implemented. Although details of the simulation strategy are described elsewhere [8], it is important to note that the characteristics of the beam-beam deflection are affected by the initial state of the machine and thus the optimal strategies for feedback design must include consideration of these effects. For these simulations, only the main linac and beam delivery system (BDS) were considered. The BDS was treated as perfect (i.e. with no initial static errors). To streamline the calculations, alignment procedures were modeled with a simplified method which should mimic the behavior of the complex algorithms. The simulation included RMS position errors of the BPMs and the accelerating structures and tilts of the structures. Structure misalignments create emittance growth mainly due to wakefields, while misalignment of the BPMs leads to dispersive emittance growth. A simple one-to-one trajectory correction was applied throughout the linac. Before entering the BDS, the mean offset and angle of the bunch were corrected.

\section{DESIGN CONSIDERATIONS FOR BEAM-BEAM FEEDBACK}

An IP feedback system is required to keep the beams in collision at the interaction point. Such a system will use the beam-beam deflection as a very precise measurement of the beam separation. The TRC design used the SLC feedback system [9] as a model, but some choices were re-evaluated in view of the likely operating regime of a future collider. Three considerations were:

- Modeling the beam-beam deflection curve.

- Optimizing the feedback deflection setpoint

- Designing the time response characteristics of the feedback controller.

\section{Modeling the Deflection Curve}

The beam-beam deflection curve in Figure 1 is a plot of the deflection of one of the beams, as a function of the beam-beam separation at the IP. The separation is controlled by an upstream kicker or dipole corrector. The deflection curve is a nonlinear function. It may be measured by using the kicker or corrector to sweep one beam across the other, while measuring the beambeam deflection with BPMs on either side of the IP. 


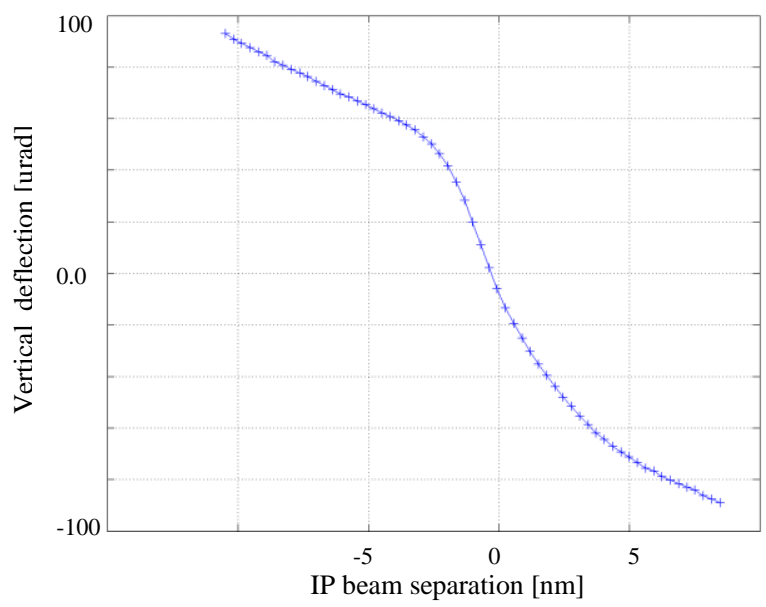

Figure 1: Beam-beam deflection curve for NLC.

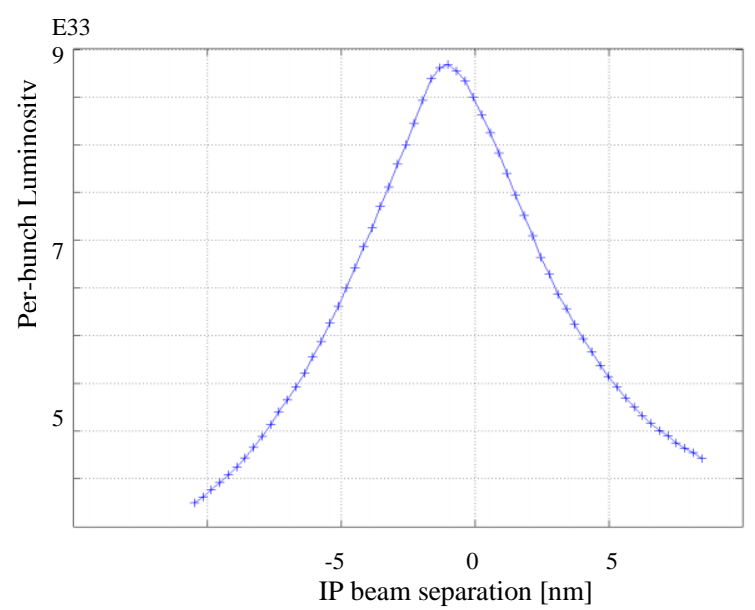

Figure 2: Luminosity as a function of beam separation for NLC

For SLC IP feedback, a linear fit was used based on the steepest slope in the central region. This resulted in a feedback response which would be conservatively slow for large beam-beam separations. For the LIAR TRC simulations, the deflection curve was modeled with a piecewise linear fit. This assumes that very good data may be available in a real collider even in the presence of beam jitter. This kind of "clean" beam-beam scan may be obtained if the scan can be performed over a single bunch train with very fast kickers. In PLACET the feedback assumed a linear deflection curve. Simulations indicate that the piecewise linear fit may not be necessary and that the simpler linear algorithm may be equally effective (see "Optimization of Feedback" and [8]).

\section{Optimizing the Deflection Setpoint}

Because the bunch shape may be asymmetric, the maximum luminosity may not be achieved when the beams are colliding head-on, as shown in Figure 2. It is preferable to find a feedback deflection setpoint which maximizes the luminosity. In the SLC, the deflection setpoint was obtained from head-on collisions as determined by the beam-beam deflection fit, regardless of luminosity. In PLACET an optimization routine used local quadratic approximations. In the LIAR TRC simulations, an asymmetric Gaussian was fit to the luminosity versus position. This might assume the existence of a fast and accurate luminosity monitor that is proportional to luminosity or is at least maximized with maximum luminosity, see [8]. However, in operation, it is possible to average many pulses to reduce the effects of monitor noise and beam jitter, or the setpoint itself may be optimized in real-time as described later.

\section{Time Response for Feedback}

In additional to optimal time response, there are other considerations in designing a beam-based feedback controller. Response speed must be faster than or comparable to what a typical operator might be able to stabilize manually, or the operator will simply take control. Robustness during changing machine conditions is also important. The SLC system [9] was designed using modern optimal control theory. This method minimized the RMS of the beam state under given assumptions, but was tuned to also give a good response for a step function. The feedback exhibited an exponential response with a time constant of about 6 feedback iterations (figure 3,-+). For the SLC IP feedback, the controller had a faster time response (figure 3,--), with additional complexities. The control system included latency in BPM processing, computing and actuation, so that the feedback was unable to implement any response to a change within the next $120-\mathrm{Hz}$ pulse. This was modeled using PredictorCorrector formalism, but the feedback response function was adversely affected. For future colliders, it is assumed that actuation would be effective on the next machine pulse, resulting in an improved feedback response function (figures 3,4, -o).

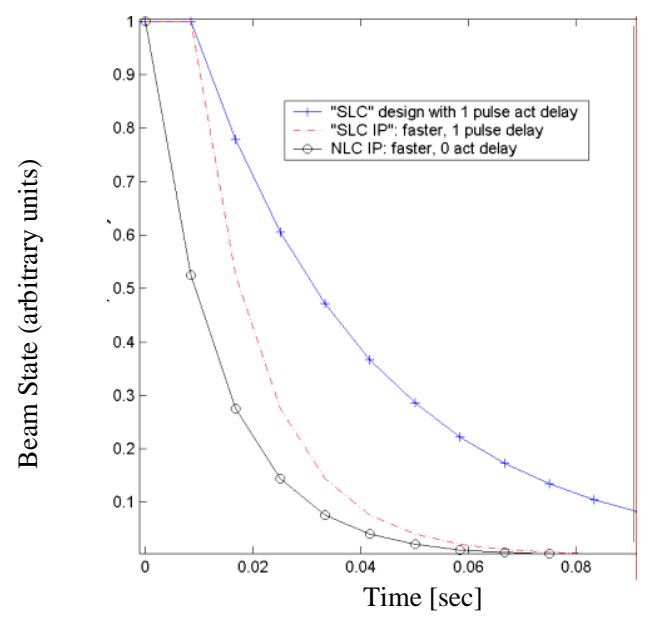

Figure 3: Step response for different feedback assumptions. 


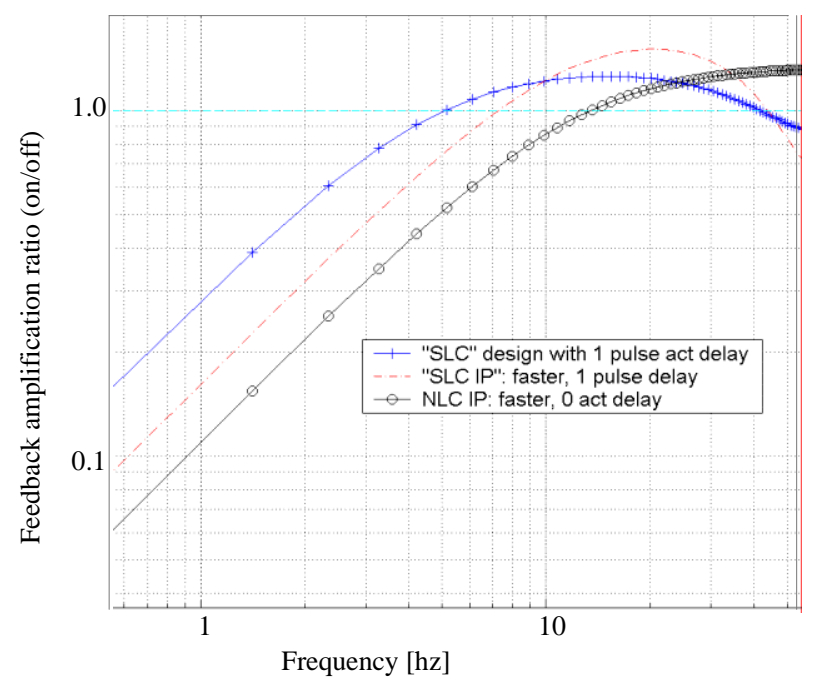

Figure 4: Feedback frequency response function for different assumptions (120-Hz pulse rate). The "NLC" design (-o), with low latency and faster response, has good response without excessive amplification.

\section{OPTIMIZATION OF FEEDBACK}

In addition to the TRC simulations, a separate effort was made to understand the sensitivity of the NLC IP feedback performance to different parameters and to implement a design which could maximize luminosity in the presence of beam jitter and other imperfections. Three parameters were adjusted in simulated-realtime:

- Slope for linear deflection approximation

- Deflection Setpoint

- Exponential time constant for feedback response.

The approach was to simulate operation over many pulses with ground motion while adjusting values of these parameters, to achieve maximum luminosity. The first two parameters were readily optimized within the framework of the TRC simulations. However the "real-time" optimization process can produce a different result from the scan procedure. The LIARbased TRC simulations assumed a perfect "instant" measure of the beam-beam deflection curve and luminosity curve. With very large ground motion, the beam shape itself can change on short timescales. Furthermore, for linear approximation of the deflection curve, the ideal slope depends upon the beam jitter. One might guess that a detailed piecewise linear fit could be optimal, but in simulations thus far, it was not found to be helpful. For smaller ground motion, the feedback response was not very sensitive to the deflection slope and a non-optimal feedback was easily able to keep the beams in collision. For very large ground motion, the shape of the deflection curve changed from pulse to pulse, and a linear fit based on the current average beam conditions worked as well or better than the detailed nonlinear fit. Adjusting both the linear slope and the deflection setpoint using timeaveraged luminosity is particularly suitable when beam jitter and measurement errors are an issue, because appropriate averaging of many pulses can be adjusted as needed.

Optimizing the exponential time constant presented additional complexities in the SLC framework because a number of matrices incorporated the time response. Therefore the algorithm was converted to an equivalent exponential form, in which the time response could be optimized by adjusting just one parameter: exponential weight. The response was nearly identical, with one exception: the SLC feedback had a small DC offset by design, and in the exponential form the DC offset was gone - a beneficial side-effect. The equations are:

state_vec $=$ expected_change +

weight * (state_vec - raw_state_vec) + raw_state_vec

delta_act $=-$ nmpt $*$ state_vec

act_vec $=$ act_vec + delta_act

expected_change $=$ bmpt $*$ delta_act

Where: weight $=$ the exponential gain: $\exp (-1 /$ npulses $)$

state_vec $=$ estimated state vector

raw_state_vec $=$ measured deflections, converted to

corrector units

act_vec $=$ actuator vector (correctors)

nmpt,bmpt are transport matrices

In the PLACET simulations a linear deflection curve was assumed and the gain was optimized for a set of 25 different machines. With these more pessimistic but also more robust assumptions the feedback performance was still very comparable to the LIARbased TRC results.

\section{FUTURE WORK}

The NLC feedback optimization studies did not include the effects of final doublet vibration and stabilization, and these are likely to affect the results. Future work will incorporate IP feedback systems with linac and BDS stabilization and steering systems, with more realistic and detailed operational scenarios.

\section{REFERENCES}

[1] "Second ILC-TRC Report", SLAC-R-606,2003.

[2] A. Seryi, et al., "Effects of Dynamic Misalignments and Feedback Performance on Luminosity Stability in Linear Colliders", in these proceedings.

[3] P. Tenenbaum, et al., "Recent Developments in the LIAR Simulation Code",SLAC-PUB-9263 (2002).

[4] D. Schulte, "Beam-Beam Simulations with Guinea-Pig", CERN-PS-99-014; ICAP 1998.

[5] http://www.slac.stanford.edu/ seryi/gm/model

[6] J. Frisch, et al., "Active Vibration R+D for the Next Linear Collider", SLAC-PUB-9419, PAC 2001.

[7] D. Schulte, et al., "Simulation Package based on PLACET. CLIC-Note 482 (2001).

[8] D. Schulte, "Luminosity in Future Linear Colliders in the Presence of Static Wakefield Effects in the Main Linac", CLIC Note 544, CERN, 2003.

[9] L. Hendrickson, et al., "Feedback Systems for Linear Colliders", SLAC-PUB-8055; PAC 1999. 